Proc. Indian Acad. Sci. (Chem. Sci.). Vol. 98, No. 3, March 1987, pp. 171-176.

(C) Printed in India

\title{
Quantitative estimation of substances spotted on filter paper by photoacoustic spectroscopy ${ }^{+}$
}

\author{
T SOMASUNDARAM, SANJAY S R RAO $†$ and P GANGULY* \\ Solid State and Structural Chemistry Unit. Indian Institute of Science, Bangalore 560012 , \\ India \\ †Department of Zoology. University of Poona, Pune 411007 , India \\ MS received 8 January 1987
}

\begin{abstract}
Quantitative estimation of coloured as well as colourless substances spotted on filter paper has been carried out by means of photoacoustic spectroscopy. The technique should be specially useful in the estimation of biological substances.
\end{abstract}

Keywords. Photoacoustic spectroscopy: quantitative estimation of spots on filter papers.

\section{Introduction}

Advantages of photoacoustic spectroscopy (PAS) in the study of materials, normally inaccessible to conventional optical spectroscopic techniques, have been described by various workers in the last few years (Rosencwaig 1980; Ganguly and Rao 1981; Tam 1986). Opaque and light scattering materials such as powders have been studied by Rosencwaig (1975). Adams et al (1980) have studied the quantum efficiency of solid fluorescent materials. Phase transition studies have been carried out by Florian et al (1978) and Somasundaram et al (1986a). Recently, Jagannathan et al (1982) have shown that PAS can be used for the study of surface acidities of solid oxide catalysts. Busse and Orgaback (1980) have used PA effect for depth profiling studies. Even biologically important materials have been studied by PAS. For example, Cahen et al (1978) have studied photosynthesis of chloroplast membranes; Balasubramanian et al (1984) have studied malaria parasites; Somasundaram et al (1986b) have studied the pigments in the thermophilic fungi.

The technique has been used for qualitative estimation of substances adsorbed on thin layer chromatograms by Rosencwaig (1975). Achwal et al (1984), however, have carried out quantitative estimation of 5-methylcytosine present in the DNA of Drosophila melanogaster by making use of a sensitive immunochemical assay and PA spectroscopy. These authors have used nitrocellulose paper for spotting the DNA and analysed the spectra for estimating the amount present. We considered it most worthwhile to investigate whether PAS was suitable for the quantitative estimation of substances spotted on ordinary filter papers, since a successful demonstration of such an application would open up a new dimension in analytical methods, especially in the case of biological substances. Estimation of substances spotted on filter paper would also serve to demonstrate one of the quintessential advantages of PA spectroscopy.

\footnotetext{
+ Contribution No. 378 from the Solid State and Structural Chemistry Unit

* To whom all correspondence should be addressed.
} 


\section{Experimental}

A single beam PA spectrometer described earlier elsewhere by Ganguly and Rao (1981) was used for this study. The light source was a $450 \mathrm{~W}$ high pressure xenon lamp. The light beam was modulated by a mechanical chopper and focussed into the cell by an adjustable mirror so that various areas of the sample could be investigated. For all the spectra obtained the conditions were as follows: scanning speed $100 \mathrm{~nm} / \mathrm{min}$; spectral resolution $20 \mathrm{~nm}$; and chopping frequency $37 \mathrm{~Hz}$. The sample holder was modified in such a way as to accommodate filter papers without

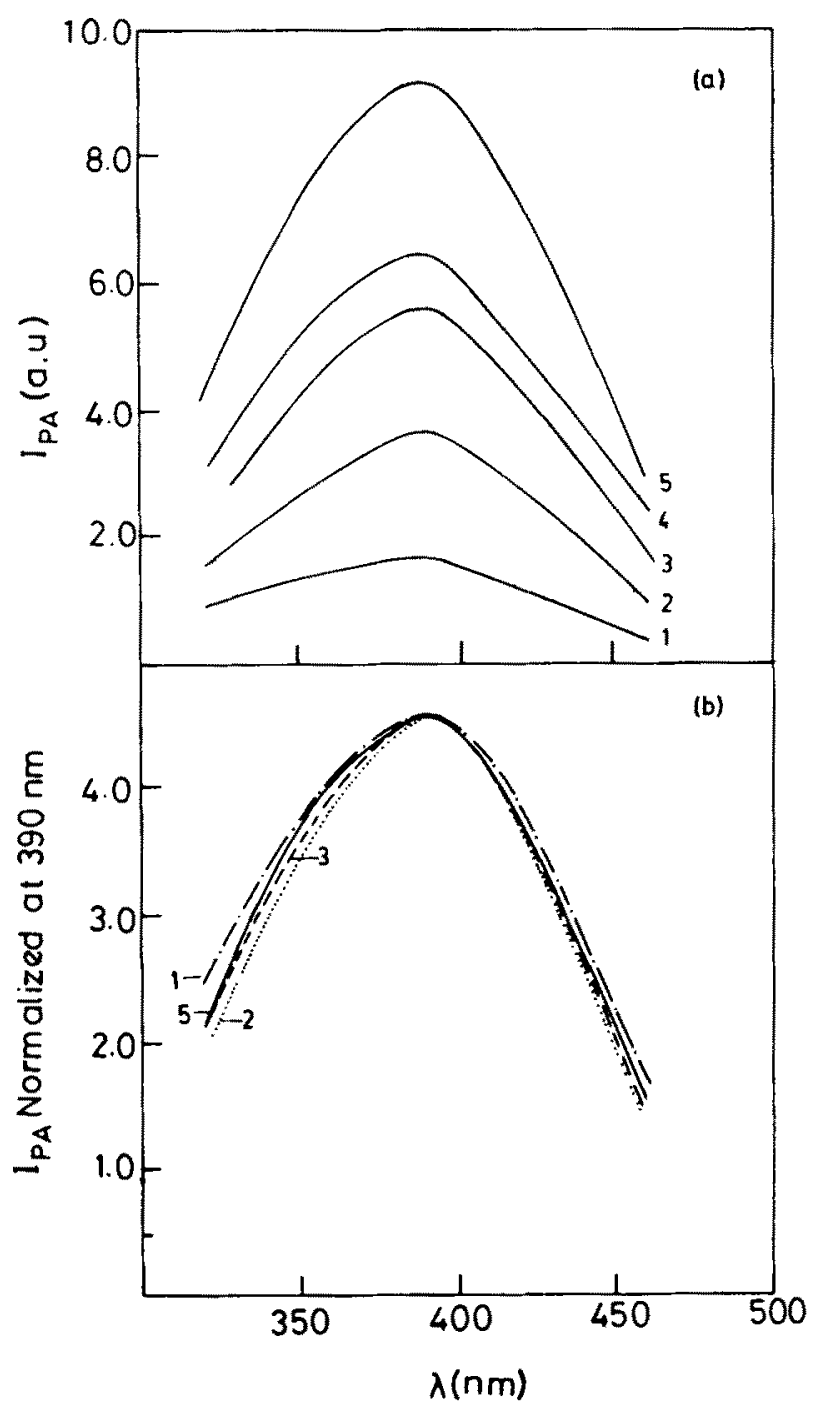

Figure 1. a) Normalized PA spectra of different amounts of $p$-nitroaniline spotted on Whatman-41 filter paper. 1: $1 \times 10^{-3} \mathrm{M} ; 2: 2.5 \times 10^{3} \mathrm{M} ; 3: 5.0 \times 10^{-3} \mathrm{M} ; 4: 7.5 \times 10^{-3} \mathrm{M} ; 5$ : $1 \times 10^{-2} \mathrm{M}$. b) Same spectra after equalizing intensities at $390 \mathrm{~nm}$. 
appreciable loss in signal. As usual normalization of recorded spectra were carried out using carbon black power spectrum.

Filter papers used were Whatman-40, 42 and 41 . The filter paper was spotted using a micro syringe using either aqueous or alcoholic solutions of the substances. The diameter of the spots did not exceed $3 \mathrm{~mm}$ (illumination covered the spots fully). Different concentrations of solutions were used for spotting different amounts, keeping the quantity of liquid spotted the same for one series of experiments (either 2 or $5 \mu \mathrm{l}$ ). Different volumes of the solutions of the same substance, containing the same weight of substance were also spotted to see whether there was any volume effect. For $p$-nitroaniline and other UV absorbing species, spotting was done either under Uv light or on cut-out pieces of filter paper. Blank filter paper pieces were used to record background contribution and they were subtracted from the signal at every wavelength before reporting. The compounds studied were $p$-nitroaniline, crystal violet, neutral red, cystosine and L-tyrosine.

\section{Results}

In figure 1a we show the carbon black normalized PA spectra of $p$-nitroaniline at various concentrations spotted on Whatman-41 filter paper after subtracting background signals due to the filter paper. After equalizing the intensities of various spectra at $390 \mathrm{~nm}$ (corresponding to various concentrations) the same spectra are replotted in figure $1 \mathrm{~b}$. One sees that all the spectra are superimposable. Similar results are obtained for the substances studied and the normalized PA spectra of all of them are shown in figure 2 .

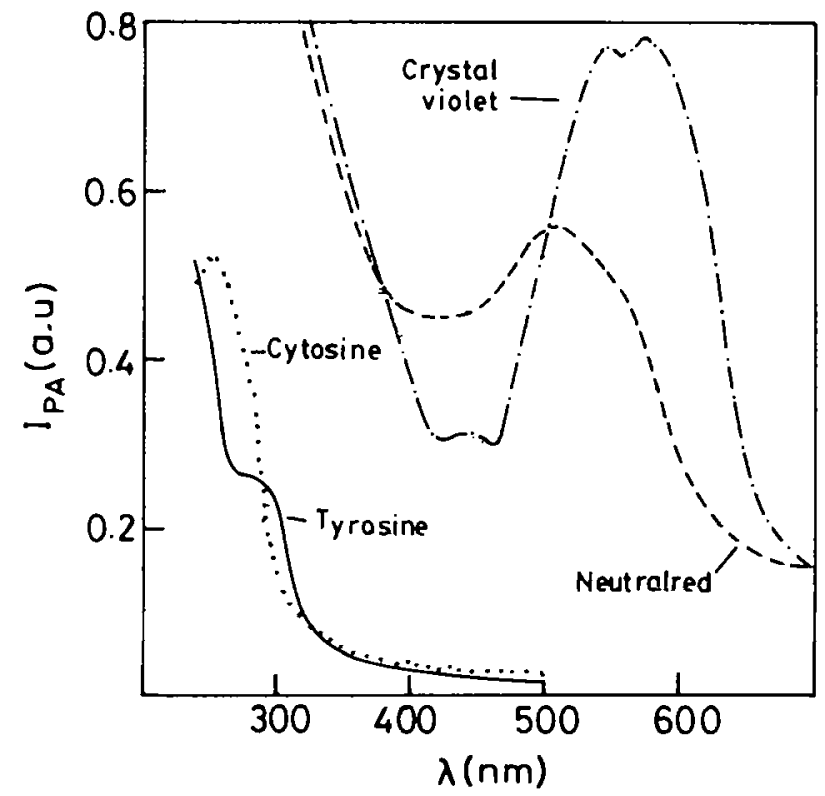

Figure 2. Normalized PA spectra of some compounds spotted on Whatman-41 filter paper. 


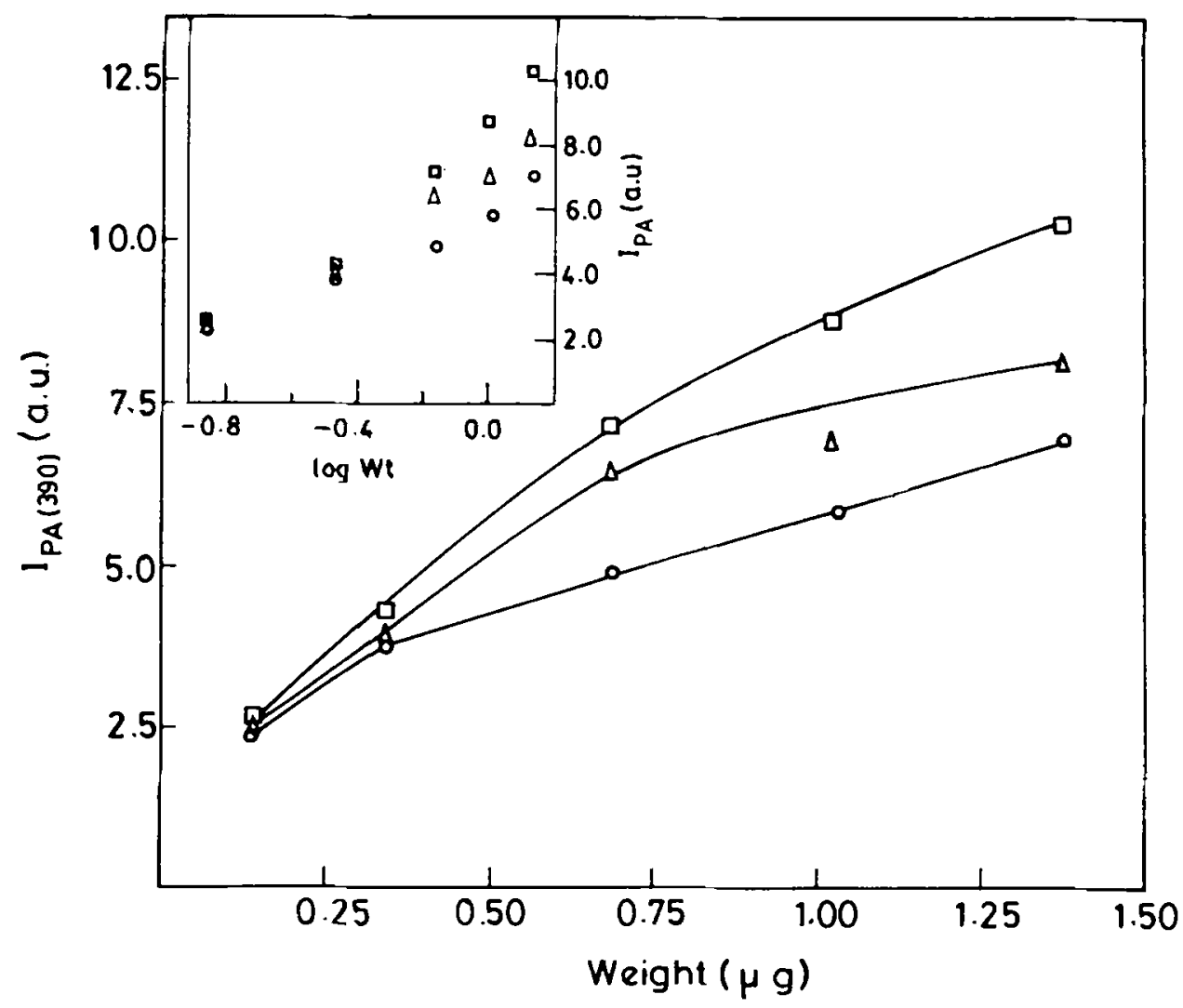

Figure 3. $I_{\mathrm{PA}}$ vs weight plot of $p$-nitroaniline spotted on three different filter papers. Circles, Whatman-41; triangles, Whatman-40; squares, Whatman-42. Inset shows $I_{\mathrm{PA}}$ vs $\log$ weight plots of the same spots.

In figure 3 we show the variation of PA intensity $\left(I_{\mathrm{PA}}\right)$ of $p$-nitroaniline at $390 \mathrm{~nm}$ for various amounts spotted on three different types of filter paper. We note that the intensities are not linearly related to the concentrations. Secondly, the intensity is greater when the filter paper is of finer grain size. For coarse grain filter paper, $I_{\mathrm{PA}}$ vs weight (wt.) of the substance, the plot is nearly linear in the high concentration regime. In figure 4 we show the variation of $I_{\mathrm{PA}}$ vs. wt. for other substances spotted on Whatman-41 filter paper. One sees a linear relationship between the intensity and concentration.

\section{Discussion}

The present study shows that there is a definite proportionality of $I_{\mathrm{PA}}$ to the wt. of the substance spotted on filter paper. To quantify this relationship, PA spectra has to be checked for saturation effects, as otherwise, increase in the amount may not bring about an increase in $I_{\mathrm{PA}}$. It is seen from figure $1 \mathrm{~b}$ that all the spectra are superimposable on one another indicating that even at the highest concentration studied there is no sign of saturation; furthermore, background subtraction 


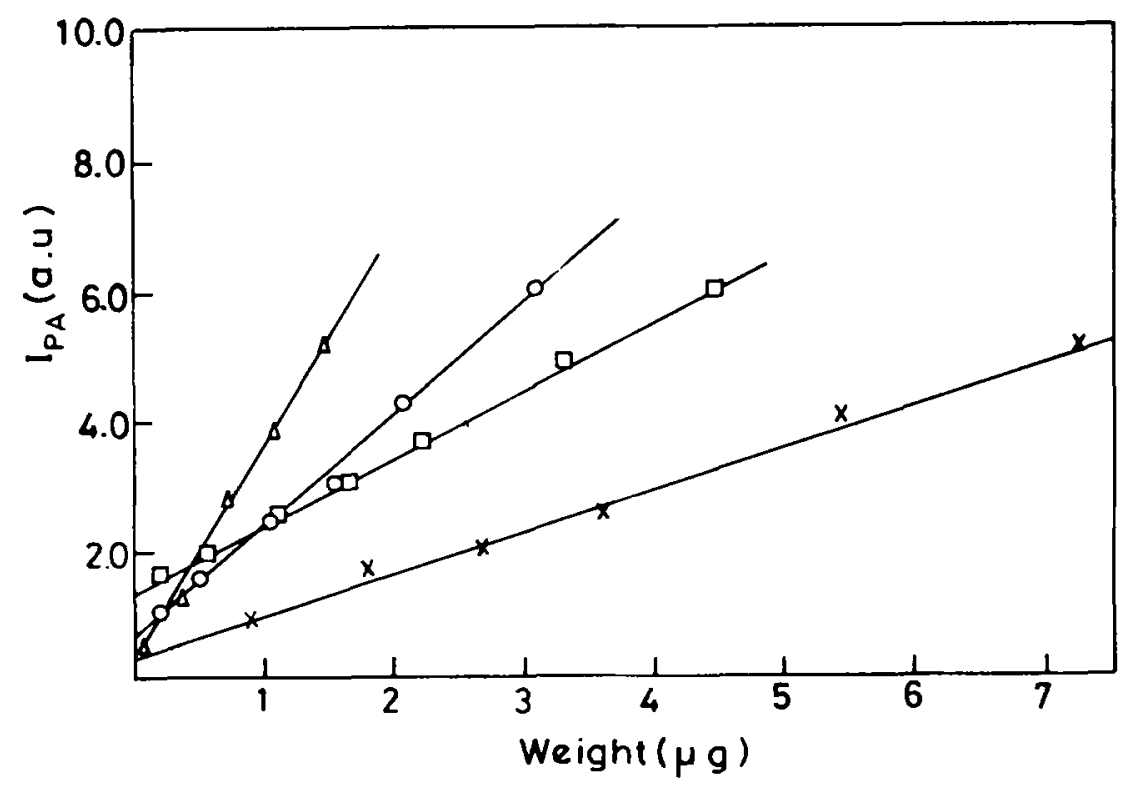

Figure 4. $I_{P A}$ vs weight of various substances spotted on Whatman-41 filter paper at the absorption maxima of the unnormalized spectra. Circles, crystal violet $(580 \mathrm{~nm})$; triangles, neutral red $(520 \mathrm{~nm})$; squares, cytosine $(280 \mathrm{~nm})$; crosses, L-tyrosine $(290 \mathrm{~nm})$.

does not result in any artifacts. This indicates that one can correlate $I_{\mathrm{PA}}$ with concentration upto a concentration range. Normalized PA spectra shown in figure 2 resemble that of standard spectra of these substances showing that in the present case the spectra have not changed significantly even under conditions of adsorption. With a proper calibration curve we can, therefore, use PAS for the quantitative estimation of substances spotted on filter paper.

The nearly linear dependence obtained with Whatman-41 filter paper (figure 4) may be exploited to advantage in the sense that the number of calibration points is reduced. This technique will undoubtedly be of great value in biology where quantitative estimation of substances spotted on filter paper often poses difficulties. Another iniportant feature is the minimum detectable amount of the substances spotted, which in our case is of the order of a few hundred nanograms. This can be gainfully used to estimate materials which are present in very small amounts and/or

The non-linear dependence of $I_{\mathrm{PA}}$ on wt. (figure 3 ) observed is likely to be due to particle size and light scattering effects. This has been discussed, in the case of powder, in some detail by Monahan and Nolle (1977) and recently by Monchalin $e t$ al (1984). Similar non-linear behaviour of the amount of light absorbed by paper containing various dyes has been pointed out by Foote (1939) and analysed by Van den Akker (1968). These authors have found, in the case of dyes adsorbed on papers, that with decreasing amounts of the dye the scattering increases. This may explain the non-linear part in the low concentration region. Since background contribution to the signal can vary with the size of the dye spot, one has to exercise caution while spotting. Spots, where weight of substance spotted is held constant but volume varied, did not show any difference in $I_{\mathrm{PA}}$. One can clearly see 
that only the solvent front advances, leaving the same sized spot in all the cases irrespective of the volume used. In our case where substances are spotted on filter paper, the absorbing chromophore is present as a thin layer on a substrate which is transparent to the radiation employed. Little is known about the generation of PA signals in such systems where the thermal properties of the substrate dominate the amplitude of the PA signal even though it is transparent to radiation (and hence inherently PA inactive). Systems such as these like solid catalysts, thin-layer chromatograms, substances adsorbed on filter papers have not been completely understood either from the theoretical or the experimental point of view. We are engaged at present in such studies.

The fraction of light absorbed seems to roughly show a logarithmic dependence on absorption coefficient, $\beta$. Melamed (1963) proposed a theory of scattering of light by particulate matter, from which the fraction of light absorption seems to show a logarithmic dependence on $\beta$ for a considerable range of $\beta \delta\left(10^{-3} \leqslant\right.$ $\beta \delta \leqslant 10^{\circ}$ ), where $\delta$ is the particle diameter. We do indeed find a nearly linear logarithmic dependence between $I_{\mathrm{PA}}$ and $\log w \mathrm{t}$. as shown in the inset of figure 3 . This dependence could be usefully employed for the purpose of calibration and estimation.

\section{Acknowledgements}

Authors thank Professor C N R Rao, FRS, for suggesting the problem and useful discussions. One of us (TS) is thankful to the CSIR, New Delhi, for a fellowship.

\section{References}

Achwal C W, Ganguly P and Chandra H S 1984 EMBO J. 3263

Adams M J, Highfield J G and Kirkbright G F 1980 Anal. Chem. 521260

Balasubramanian D, Mohan Rao Ch and Banijpan B 1984 Science 223828

Busse G and Orgaback A $1980 \mathrm{~J}$. Appl. Phys. 513576

Cahen D, Malkin S and Lerner E I 1978 FEBS Lett. 91339

Florian R, Pelzl J, Rosenberg M, Vargas H and Wernhardt R 1978 Phys. Status Solidi A48 K35

Foote W J 1939 Tech. Assoc. Papers (TAAPI) Series xxii, p. 397

Ganguly P and Rao C N R 1981 Proc. Indian Acad. Sci. (Chem. Sci.) 90153

Jagannathan K, Ganguly P and Rao C N R 1982 J. Catal. 75262

Melamed N T 1963 J. Appl. Phys. 34560

Monahan Jr. E M and Nolle A W 1977 J. Appl. Phys. 483519

Monchalin J.P, Bertrand L, Rousset G and Lepourte F 1984 J. Appl. Phys. 56190

Rosencwaig A 1975 Anal. Chem. 47592 A

Rosencwaig A 1980 in Pholoacoustics and photoacoustic spectroscopy: Chemical analysis Vol: 57 (New York: John Wiley)

Somasundaram T, Ganguly P and Rao C N R 1986a J. Phys. C19 2137

Somasundaram I, Rao S S R and Maheshwari R 1986b Curr. Sci. 55957

Tam A C 1986 Rev. Mod. Phys. 58381

Van den Akker 1968 in Modern aspects of reflectance spectroscopy (ed.) W W Wendlandt (New York: Plenum) p. 27 